\title{
Measurement of viscous sound absorption at 50-150 kHz in a model turbid environment
}

\author{
Niven R. Brown and Timothy G. Leighton \\ Institute of Sound and Vibration Research, University of Southampton, Southampton SO17 1BJ, \\ United Kingdom \\ Simon D. Richards \\ Defence Evaluation and Research Agency, DERA Winfrith, Winfrith Technology Centre, Dorset DT2 8XJ, \\ United Kingdom \\ Anthony D. Heathershaw \\ Defence Evaluation and Research Agency, DERA Unit, Southampton Oceanography Centre, Southampton \\ SO14 3ZH, United Kingdom
}

(Received 26 January 1998; accepted for publication 25 June 1998)

\begin{abstract}
The visco-thermal absorption of sound by suspended particulate matter can be reliably measured using a reverberation technique. This absorption may have an adverse effect on the performance of sonars operating at $50-300 \mathrm{kHz}$ in coastal waters where suspensions are often present in significant concentrations. A series of experiments has been performed to study the viscous absorption by suspensions in the frequency range of $50-150 \mathrm{kHz}$. In the test volumes employed, the effect is small. It is therefore measured by taking the difference in reverberation times of a volume of water with and without particles. This greatly reduces the effect on the measurement of the other sources of absorption. Even so, it is necessary to design the experiment to characterize and minimize acoustic losses which occur at the surfaces of the container, the hydrophones, and their cables, and losses associated with bubbles and turbulence. These effects are discussed and results for particulate absorption for suspensions of spherical glass beads are presented and compared to theoretical predictions. Measured absorption agrees well with that predicted by theory for concentrations above $0.5 \mathrm{~kg} / \mathrm{m}^{3}$ and up to $2.0 \mathrm{~kg} / \mathrm{m}^{3}$. [S0001-4966(98)01610-5]
\end{abstract}

PACS numbers: 43.30.Es, 43.35.Bf [DLB]

\section{INTRODUCTION}

The acoustic absorption properties of suspended particulate matter in natural bodies of water are not well characterized, although there are a number of applications (e.g., naval mine-hunting sonars, acoustic Doppler current profilers) where such knowledge would be important, particularly in shallow water in the frequency range 50-300 kHz. Typical suspensions contain particles in the size range 1-100 $\mu \mathrm{m}$ where a variety of shapes and concentrations from $0.1 \mathrm{~kg} / \mathrm{m}^{3}$ up to $4 \mathrm{~kg} / \mathrm{m}^{3}$ are possible. They are liable to produce significant absorption losses. ${ }^{1}$ There may also be the potential for flocculation and turbulence. The acoustic absorption of such systems is not known and, if models or inversion procedures are to be successfully implemented, must be quantified. $^{2}$

There are many potential contributory factors to signal loss in the water column. Most of the work concerning suspensions has focused on scattering. Absorption from certain phenomena other than particles is, by comparison with particulate absorption, well understood. Within the water column, temperature, salinity, pressure, and the concentrations of absorbed gas may vary, affecting the overall acoustic absorption. ${ }^{3}$ If bubbles are present, they may contribute significantly to the loss of acoustic energy through thermal and viscous effects, and also through acoustic re-radiation. ${ }^{4,5}$ It is possible to incorporate such factors individually into a description of the acoustic absorption. For deployments in the environment in question, however, it may not be sufficient simply to quantify the contribution from the suspended particulate matter: The possibility of synergy between these factors should be explored. There is, for example, an association between suspended particulate matter and the stabilisation of gas pockets. ${ }^{6}$

This paper presents results from an experimental study which aims to quantify the viscous absorption associated with suspended particulate matter. Recent theoretical descriptions of the phenomenon have been published by two of the authors, ${ }^{1,7}$ and these are compared here with experimental measurements. The viscous absorption effects of the suspensions, once determined, can be incorporated into acoustic propagation models.

\section{THEORY}

The theory for visco-thermal attenuation by particles is well established and has been presented in some detail previously. ${ }^{1,7}$ A brief synopsis is given here for clarity. Sound propagating in seawater is attenuated via a number of mechanisms such that the intensity, $I$, after propagation over range, $r$, is given by

$$
I=I_{0} e^{-2 \alpha r},
$$

where $\alpha$ is the volume attenuation coefficient of the seawater. In this equation $\alpha$ is in units of Nepers $/ \mathrm{m}$ but units of $\mathrm{dB} / \mathrm{m}$ have been used in the remainder of the paper. The total 
attenuation is the sum of the attenuation due to clear seawater, $\alpha_{w}$, and that due to scattering and viscous absorption by the suspended sediment, $\alpha_{s}$ and $\alpha_{\nu}$, respectively, i.e.,

$$
\alpha=\alpha_{w}+\alpha_{s}+\alpha_{\nu} .
$$

Sound absorption in clear seawater is itself the sum of absorption due to pure water and ionic relaxation processes involving boric acid and magnesium sulphate. One expression for the seawater absorption term commonly employed is given by Fisher and Simmons, ${ }^{8}$ which was derived from laboratory data using Lyman and Fleming artificial seawater. ${ }^{9}$ A more recent and arguably more complete expression is given by Francois and Garrison. ${ }^{3}$ The boric acid relaxation frequency is $O\{1 \mathrm{kHz}\}$, while that for magnesium sulphate is $O\{100 \mathrm{kHz}\}$. Both are temperature and $p \mathrm{H}$ dependent. For the frequency range of interest in this study only magnesium sulphate would make a significant contribution to the total attenuation and its behavior is well documented. Since the experimental protocol involved minimizing as far as practicable sources of absorption other than particles, the water used in the current study was filtered and degased.

Attenuation from scattering is due to sound energy being reflected and diffracted from the main propagation path by the suspended particles. A number of models for scattering exist. The scatterers can be modeled as homogeneous spheres, which may be rigid and movable, rigid and immovable, or elastic. ${ }^{10,11}$ From the point of view of a closed reverberation volume, however, as is used experimentally here, scattering does not produce an attenuation per se. This is because, if the walls are perfectly reflecting, the acoustic energy would remain within the volume and continue to be attenuated by other loss mechanisms.

The method of attenuation of interest here is that due to viscous absorption which occurs in the viscous boundary layer surrounding the particles. The boundary layer is generated because the acoustic wave causes out-of-phase movement between the fluid and the particle which creates a velocity difference between the two. Unlike scattering, where the acoustic impedance mismatch at the particle surface is of importance, viscous absorption is an inertial effect governed by the density difference between the fluid and particle. Because of this, it is not possible to use neutrally bouyant particles as they would simply move in-phase with the fluid and, thus, create no viscous boundary layer. Using Urick's ${ }^{12}$ expression for the viscous absorption coefficient, and taking the attenuation to be constant along the path length and assuming all particles are the same size, the attenuation coefficient due to viscous absorption may be expressed as

$$
\alpha_{\nu}=\left(10 \log e^{2}\right)\left(\frac{\epsilon k(\sigma-1)^{2}}{2}\left[\frac{s}{s^{2}+(\sigma+\delta)^{2}}\right]\right) \mathrm{dB} / \mathrm{m},
$$

where

$$
\begin{aligned}
& \delta=\frac{1}{2}\left[1+\frac{9}{2 \beta a}\right], \\
& s=\frac{9}{4 \beta a}\left[1+\frac{1}{\beta a}\right],
\end{aligned}
$$

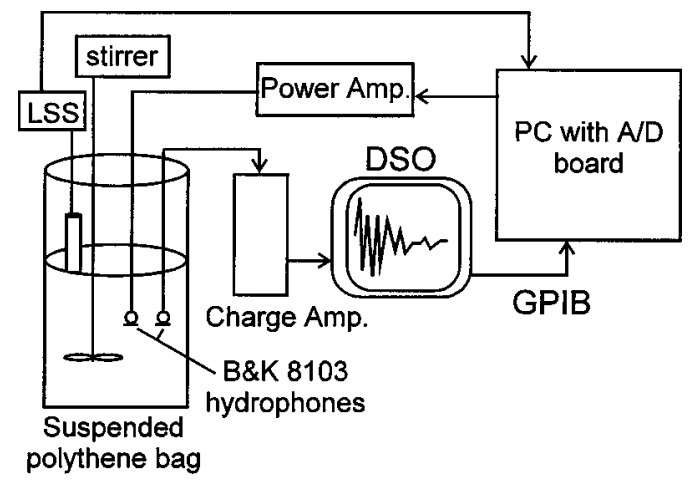

FIG. 1. Schematic diagram of the experimental apparatus.

$\sigma=\rho_{s} / \rho_{0}, \beta=\sqrt{\omega / 2 \nu}$ is the reciprocal of the viscous skin depth, $\rho_{s}$ and $\rho_{0}$ are the densities of the particulate and fluid, respectively, $\nu$ is the kinematic viscosity of the ambient fluid, $\epsilon$ is the volume concentration of particulate, $a$ is the particle radius, $k$ is the acoustic wave number, and $\omega$ is the angular frequency of the incident pressure wave. The first term on the right-hand side of Eq. (3) is a constant which converts attenuation from Nepers/m to $\mathrm{dB} / \mathrm{m}$.

In this theory the assumption is made that the attenuation depends linearly on sediment concentration. Urick ${ }^{12}$ showed that this linear dependence is valid up to volume concentrations of about $8 \%-9 \%$. Below this concentration the suspension may be considered to be dilute, meaning that the effects of particle interaction, such as multiple scattering, may be ignored. The maximum mass concentration considered in this paper is $2 \mathrm{~kg} / \mathrm{m}^{3}$, which for quartz particles corresponds to a volume concentration of about $0.08 \%$. The suspensions may therefore be considered to be dilute and the assumption of linear dependence on concentration is taken to be valid.

\section{EXPERIMENTAL METHOD}

The use of reverberation time to determine the attenuating characteristics of fluids has been credited by Kurtze and Tamm ${ }^{13}$ to the work of Meyer and Skudrzyk. Differences in decay rates for a given volume of fluid may be equated to variations in the absorptive properties of the fluid and the boundaries of the volume. Preliminary tests ${ }^{14}$ in the current study were performed in a large, thick-walled plastic tank containing approximately $0.6 \mathrm{~m}^{3}$ of water. Decay traces from this apparatus were compared to traces taken from a smaller system comprising a suspended polythene bag containing only $16 \ell$ of water. Although the ratio of surface area to volume was increased in the smaller system, the reverberation time increased, emphasizing the importance of reducing the losses at the boundaries in order to maximize the relative losses in the fluid. It should be noted here that the attenuation of pure water at $20^{\circ} \mathrm{C}$ and 1 atm is only $0.002 \mathrm{~dB} / \mathrm{m}$ at 100 $\mathrm{kHz}$ according to Fisher and Simmons. ${ }^{8}$ Clearly any reduction in the boundary losses will greatly improve the estimation of the fluid losses.

The system used is shown in schematic form in Fig. 1. The signal generation, data acquisition, and signal processing are controlled by a personal computer running LABVIEW soft- 
ware. The output signal is sent to a power amplifier and then to a Brüel \& Kjær 8103 hydrophone. Signals are received by a second 8103 hydrophone and are monitored, after suitable amplification, by a LeCroy digital storage oscilloscope and are finally transferred to the computer via a GPIB interface for storage and analysis. The $16 \ell$ of water is contained in a thin-walled polythene bag which is supported on a suspended ring. This provides an approximation to a pressure release surface around the whole volume, thus minimizing boundary losses. A mechanical stirrer is used to lift the particulate into suspension and is removed while data are being recorded. The dynamic concentration of the suspension can be monitored using a light scattering sensor (LSS). This monitors the settling out from suspension of the particulate. The acoustic and LSS measurements are performed separately as the presence of the LSS and the stirrer in the suspension represent additional absorbing surfaces which make measurement of the particulate contribution more difficult.

To measure the reverberation time of the volume it is necessary to record the decay of a sound field as a function of time. Ideally, the reverberation time is determined from the decay of a diffuse sound field. A diffuse sound field is one where the average energy density is the same throughout the volume considered and all directions of propagation are equally probable. ${ }^{15}$ The onset of a diffuse sound field in an enclosure can be described by the Schroeder cutoff frequency. This gives an indication of the lowest frequency at which the modal density is sufficient to constitute a diffuse field. The Schroeder cutoff frequency, $f_{\text {Sch }}$, can be expressed as $^{16}$

$$
f_{\mathrm{Sch}}=\left(\frac{c^{3}}{4 \ln 10}\right)^{1 / 2}\left(\frac{T}{V}\right)^{1 / 2}
$$

where $T$ is the reverberation time of an impulsive noise source (i.e., the time for the sound pressure level to fall by 60 $\mathrm{dB}), c$ is the speed of sound in the fluid, and $V$ is the volume of the enclosure. Values of $f_{\text {Sch }}$ for the system used were between 50 and $75 \mathrm{kHz}$. This is near the lower limit of the frequency range under consideration in this project.

Two techniques have been used to generate a sound field: an impulse and a burst of uniform white noise. Both these techniques produce a broadband sound field. The advantages of a long burst are that the sound field is given time to build up to a constant level before being cut. This improves the signal-to-noise ratio. Also, because there is a more uniform sound field, the decaying sound field is less prone to large perturbations due to direct reflections and particular modes of the volume.

A typical test sequence consists of the suspension being stirred until the particulate is homogeneously spread throughout. The time for this to occur can be verified by the LSS and is of the order of a few seconds. Care must be taken to ensure that particulate does not collect in the eddies generated in the corners of the bag adjacent to the bottom seam during the stirring. Ten noise bursts are sent to the emitting hydrophone and their responses are recorded by the computer. The test sequence takes approximately $35 \mathrm{~s}$. This is about the time limit before there is a significant change in the suspended particulate concentration as measured by the LSS.

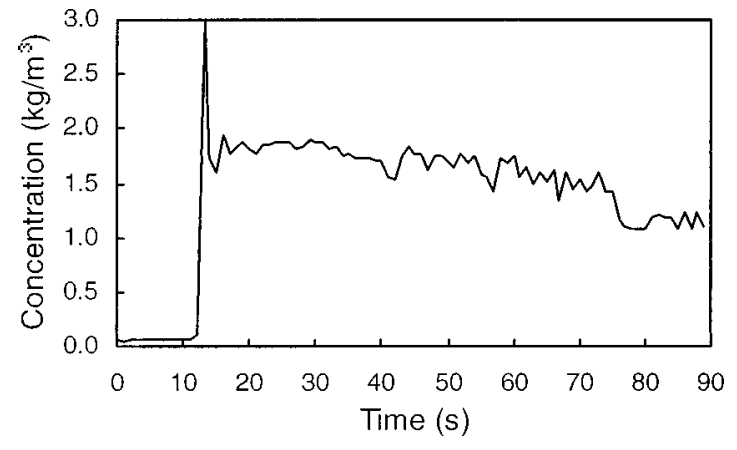

FIG. 2. Variation of suspended particulate concentration with time for a $2.0-\mathrm{kg} / \mathrm{m}^{3}$ suspension.

Figure 2 shows a typical LSS output. In this instance the stirrer was turned on at $12 \mathrm{~s}$ and off at $32 \mathrm{~s}$. The particle concentration is more accurately determined by weighing in the particulate to give the desired suspension concentrations. The LSS is simply used to verify the mixing and settling processes prior to acoustic tests being performed.

The particle size distribution was also determined independently of the acoustic tests by analyzing a sample of the particulate in a laser diffraction analyzer. This determines the volume distribution of particles over the size range, $0.4 \mu \mathrm{m}-$ $1000 \mu \mathrm{m}$. The dynamic variation of the particle size distribution cannot, however, readily be obtained by this method as a relatively substantial volume of water must be taken from the suspension. This would obviously affect the reverberation characteristics of the volume. Figure 3 shows the particle size distribution for the glass beads used in this study.

The decay rates were determined by applying the method of integrated impulse response ${ }^{17}$ (IIR) to the sound field from the time that the driving signal was cutoff. This method was used, even for signals derived from nonimpulsional sources, as it gave a smooth estimate of the decay rate. The value of the integrated impulse response represents the ensemble average of the squared noise responses at time $t$ $=t^{\prime}$ after the onset of decay which is equal to the squared tone-burst response integrated from time $t=t^{\prime}$ to $t=\infty$ or, in practice, to when the background noise level is greater than the signal of interest. The practical implementation of this method is as follows. The response of the volume to the burst of random noise (which contains the frequency range of interest) is squared, then backward integrated from an upper time limit (some time before the response is exceeded

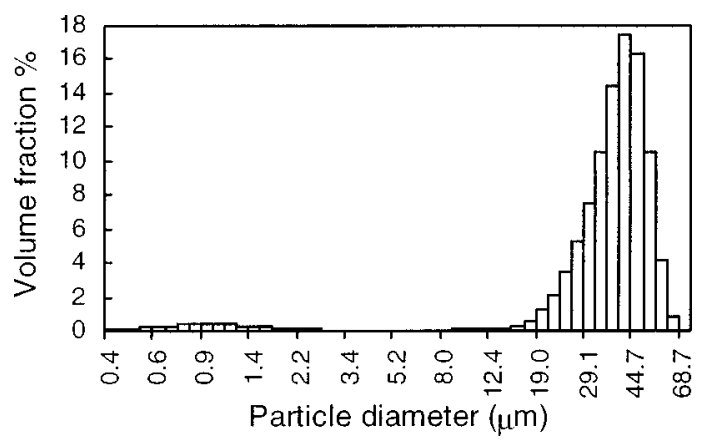

FIG. 3. Particle size distribution for glass beads. 
by the background noise) to the lower time limit when the sound burst was cut off. This produces the IIR curves shown in the results section. The slope of this curve is determined from a linear regression over the initial, linear part of the curve. Typically, the lower time limit for the linear regression was $10 \mathrm{~ms}$ after the sound was cut off (the burst lasted $20 \mathrm{~ms}$ ) and the upper limit was variable, the choice depending on the rapidity of the decay and the linearity of the response.

Post-processing of the results involved performing the IIR analysis at each of the desired frequency bands. The raw data were filtered after acquisition using a Butterworth bandpass filter in $10-\mathrm{kHz}$ bands over the frequency range 50-150 $\mathrm{kHz}$. Above this frequency, the response becomes increasingly nonlinear making it difficult to obtain an estimate for the linear decay of the sound field. The data were also reduced into time bins which represent the rms of the signal for a user-defined number of samples. This was typically 100 samples. The sampling frequency of the oscilloscope was $500 \mathrm{kHz}$ and the sample duration was $0.2 \mathrm{~s}$.

Measurements were made on particulate-free water and then on water containing varying concentrations of glass beads. Prior to experimentation, the water was passed through a reverse osmosis system, then filtered to remove any remaining particulate matter. The water was then degased under vacuum and the level of dissolved oxygen was monitored throughout the test to see what effect the addition of particles or the stirring process had. The dissolved oxygen content varied from $51 \%$ to $65 \%$ over the course of the measurements (approximately four hours). No bubbles could be detected. The glass beads have a high sphericity and are, thus, representative of the spherical particles used in the theoretical modeling. The difference in reverberation of the two systems determines the contribution of the particles to the total absorption according to the following analysis.

\section{Determination of absorption from reverberation times}

The decay of a diffuse sound field where absorption occurs at the boundary and within the propagating medium is characterized by the reverberation time, $T$, given by ${ }^{18}$

$$
T=\frac{55.3 V}{c(A+8 \alpha V)},
$$

where $A$ is the total sound absorption at the boundaries of the volume, and $\alpha$ is the attenuation coefficient of the fluid in Nepers $/ \mathrm{m}$. The quantity $A=S \bar{a}$ is expressed in units of metric sabin, $\mathrm{m}^{2}$, where $S$ is the surface area of the volume $\left(\mathrm{m}^{2}\right)$ and $\bar{a}$ is the average Sabine absorptivity (dimensionless). The first term in the brackets, $A$, represents the sound absorption at the boundaries; the second term, $8 \alpha V$, is the absorption in the medium. If $T_{w}$ and $T_{s}$ are the reverberation times of the particulate-free water and the water containing the particulate, respectively, then the difference in the attenuation coefficients of the fluids, $\Delta \alpha$, in $\mathrm{dB} / \mathrm{m}$ is given by

$$
\Delta \alpha=\left(10 \log e^{2}\right) \frac{55.3}{8 c}\left(\frac{1}{T_{s}}-\frac{1}{T_{w}}\right) .
$$

This represents the attenuation due to the addition of the particulate. This equation makes three important assumptions:

(i) that the speed of sound of the suspension stays constant as particles are added;

(ii) that the volume remains constant; and

(iii) that the addition of the particles does not affect the absorptivity of the boundaries.

The sound speed in suspensions can be calculated by using the formulation developed by Ahuja. ${ }^{19}$ Assuming a rigid particle (i.e., the particle "viscosity" is much greater than the fluid viscosity), then the change in sound speed for the suspensions considered in this work is less than $0.01 \%$. The volume fraction of a $2-\mathrm{kg} / \mathrm{m}^{3}$ suspension of glass beads having a density of $2400 \mathrm{~kg} / \mathrm{m}^{3}$ is only $0.08 \%$, so that the assumption of constant volume is reasonable. Finally, if changes in acoustic impedance are principally responsible for changes in behavior at the boundary, then the product of the change in density and change in sound speed of the particulate suspension represents an error of less than $0.1 \%$. Thus the properties of the bag can be assumed to have almost no contribution to the sound transmission at the boundary. This is reasonable to assume as the walls of the bag are thin $(0.03 \mathrm{~mm})$, certainly in terms of the wavelengths under consideration, and there is very little acoustic impedance mismatch with the water. Hence, the walls will move in phase with the water and be virtually acoustically transparent. The bag itself acts like an approximately pressure release surface and any change in behavior at the boundary will be due to changes in the properties of the fluid. There may be viscous boundary layer losses at the bag but these will be consistent between the clearwater and particulate suspension cases. These losses, along with losses due to the presence of the hydrophones, prevent a simple measurement of the absolute attenuation of the fluid as is the case for other measurement systems such as a spherical resonator, as noted by one of the early workers in that field. ${ }^{13}$

\section{RESULTS}

A series of tests was performed on water and glass bead suspensions with concentrations from 0.25 to $2.0 \mathrm{~kg} / \mathrm{m}^{3}$ in steps of $0.25 \mathrm{~kg} / \mathrm{m}^{3}$. Figure 4 shows typical binned time traces and their corresponding IIR curve at $100 \mathrm{kHz}$ for pure water and a $1.0-\mathrm{kg} / \mathrm{m}^{3}$ suspension of glass beads. The IIR curve clearly represents the decay rate of the sound energy in the volume. The $y$-axis scale is the sound pressure level (SPL) in $\mathrm{dB}$ re: $1 \mu \mathrm{Pa}$. The two curves are offset because of the processing performed to obtain the IIR curve. At this frequency there is almost a $60-\mathrm{dB}$ dynamic range. The output burst lasted $20 \mathrm{~ms}$ and the increase in the sound pressure over this time can be observed in the figures. The effect of the particulate is clearly seen. Note that for presentation purposes the time over which the IIR has been applied has been extended to cover the whole sample period, hence the tailing off of the IIR curve once the signal approaches the background noise level.

Figure 5 shows the reverberation time variation as the particulate concentration is increased. Each curve represents 
GPL (dB re $1 \mu F G)$

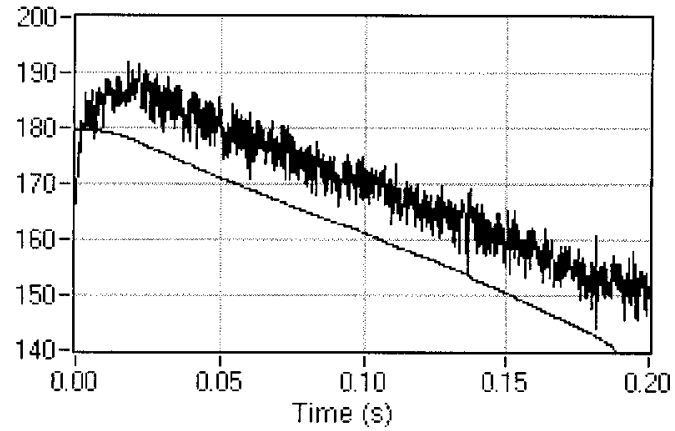

(a)

SPL (dE re $\left.1 \mu F^{\prime} a\right)$

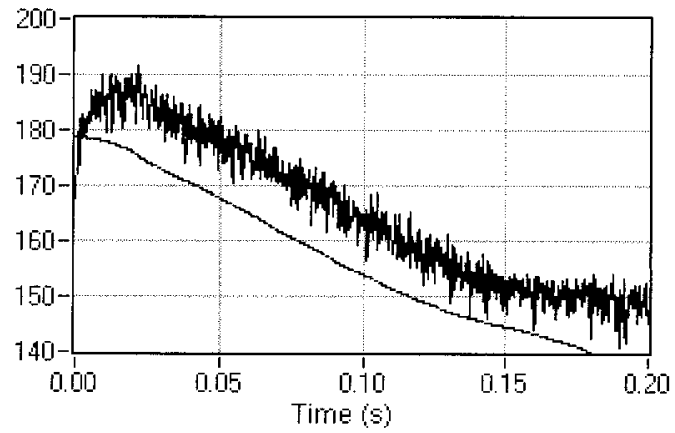

(b)

FIG. 4. Typical binned time traces at $100 \mathrm{kHz}$ for reverberation time calculation (upper trace) with their corresponding integrated impulse response curve (lower trace) for (a) pure water, and (b) a $1.0-\mathrm{kg} / \mathrm{m}^{3}$ suspension of glass beads.

the mean of three tests of ten pings each. Also shown are two curves for pure water: one for calm water and a second for stirred water. As the test suspensions containing particulate must be stirred, the stirred water response was taken as the reference signal for the subsequent calculation of the particulate attenuation. The error bars on the pure water curves represent the uncertainty in measuring the reverberation time at one point rather than throughout the volume. This is discussed fully in the following section. For clarity, only every other concentration is shown in Fig. 5. The decreasing trend

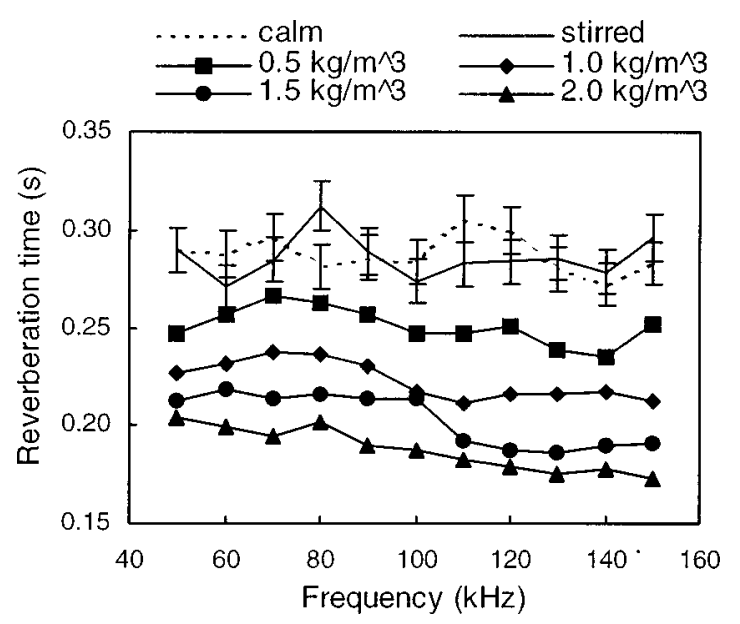

FIG. 5. Reverberation time for calm and stirred pure water and for various concentrations of glass beads. Error bars for water curves represent uncertainty due to measurement at a single location. in reverberation time with increasing particulate concentration is quite apparent.

The reverberation times are converted to changes in attenuation according to Eq. (8) and are then normalized with respect to particle concentration. The units of $\mathrm{dB} \mathrm{m}^{2} / \mathrm{kg}$ can be converted to $\mathrm{dB} / \mathrm{m}$ via multiplication by the given concentration. They can then be compared to a theoretical prediction [Eq. (3)] which has been calculated for a $1-\mathrm{kg} / \mathrm{m}^{3}$ suspension having the same particle size distribution as shown in Fig. 3. The normalized attenuation due to the particles is shown in Fig. 6 at each of the measured concentrations. For all data, the theoretical prediction lies within two standard deviations of the data, and in most cases, within one. As expected, the magnitudes of both the uncertainty and the discrepancy between the data points and theory increase at the lowest concentrations. Appreciation of the errors is extremely important in interpreting these results, and these are discussed in the next section.

\section{DISCUSSION}

The previous results show that this simple system is capable of producing reliable results for particulate suspensions once there is sufficient difference between the reference pure water signal and the particulate suspension signal. Taking a difference in this way in principle eliminates the effects of other loss mechanisms, such as the boundaries and the hydrophones. However, their effects should be minimized in order to enhance the behavior of the particulate attenuation. That is why the suspended thin-walled bag has been developed as opposed to using a solid containment vessel.

The principal difficulty of measuring the behavior of this type of suspension is maintaining the particulate in suspension. By stirring, the suspension becomes well mixed and the particulate remains suspended for a sufficiently long time to enable the measurements to be taken (see Fig. 2). However, the stirring process may affect the acoustics of the water volume, even when there is no particulate present. Acoustic energy may be absorbed by isotropic turbulence through perturbation of the turbulence field by the acoustic wave, leading to anisotropic Reynolds stress. Within the time taken for the Reynolds stress to return to isotropy, turbulent kinetic energy will have been redistributed among turbulence components as it cascades from the large scale to the dissipation scale, resulting in a net loss of energy from the acoustic wave. Noir and George ${ }^{20}$ obtained an expression for the absorption coefficient resulting from this effect which may be used to estimate the absorption as a function of the rate at which turbulent kinetic energy in the system is dissipated. Consideration of the maximum rate of kinetic energy supplied by the mechanical stirrer leads to estimates of the turbulence absorption coefficient which are $O\left\{10^{-8} \mathrm{~dB} / \mathrm{m}\right\}$, which is several orders of magnitude smaller than the measured attenuation coefficient and the predicted viscous absorption coefficient. The effect of turbulence is also several orders of magnitude smaller than the error associated with the comparison between stirred and calm water in Fig. 5. It is therefore concluded that the effect of sound absorption by turbulence may be neglected in the present system. 

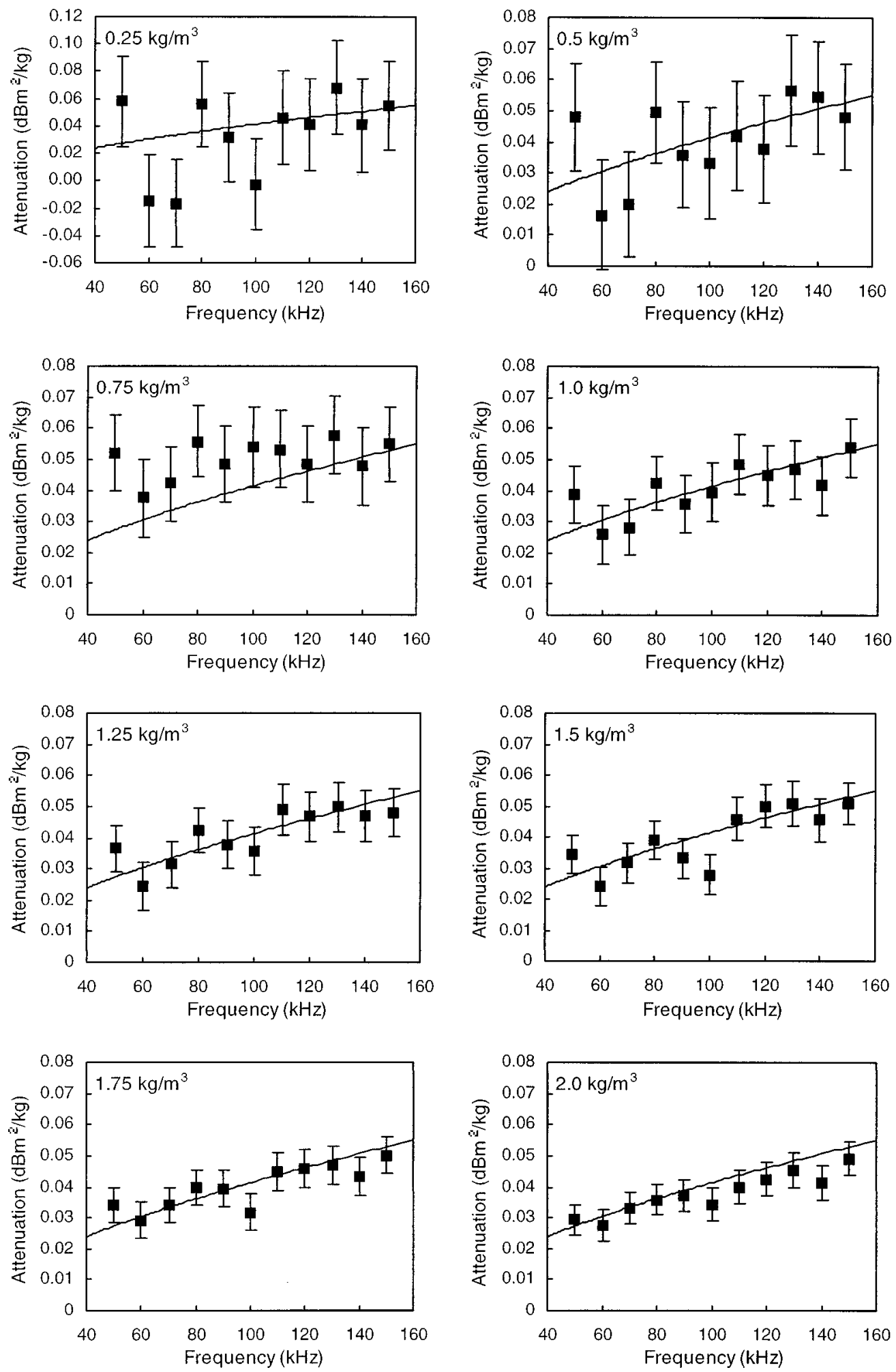

FIG. 6. Normalized particulate attenuation at the indicated concentrations compared to the prediction (solid line). Because of the normalization the prediction is always the same. An explanation of the error bars is given in Sec. IV.

Ideally the sound field would be measured at a number of places in order to obtain a spatial average throughout the bag. This would verify that the sound field was indeed diffuse and would give an indication as to how the settling process affects the attenuation. Measurements have been performed on pure water to assess the assumption of a diffuse sound field. However, the mere fact that more of the hydro- phone was inserted into the water caused a very significant change in the level of absorption. For every $10 \mathrm{~cm}$ of cable inserted the increased absorption was of the same level as that due to $1 \mathrm{~kg} / \mathrm{m}^{3}$ of the particulate used in this study. It was, therefore, not possible to perform a volume average of the sound field. Instead the sound field was measured at a number of points at the same depth so that the same amount 
of hydrophone was in the water. One standard deviation of these tests was equivalent to a $4 \%$ error in the estimation of the reverberation time if it were measured at one location rather than averaged in a plane. Error bars of $4 \%$ are shown for the calm and stirred water reverberation traces in Fig. 5. These show that the variation in reverberation due to stirring is within the range of error due to single location measuring. Other sources of error, such as the variation of the speed of sound with temperature, the estimation of the concentration based on the weight of added particulate, and the measurement of the water volume, were all significantly less than this and generally less than 1\%. The error bars in Fig. 6 are, therefore, calculated using a $4 \%$ error in the measurement of the reverberation time and the cumulative error in the estimate of the suspension concentration, even though the pingto-ping variation for reverberation measurements made at one location is less than 1\%. It is clear from Fig. 6 that as the difference in the reverberation time between the reference pure water signal and the particulate suspension signal increases, the relative error in the attenuation, even after normalization, decreases.

The data in Fig. 6 support the trend predicted by theory (i.e., absolute values of attenuation and its gradient with respect to frequency). The contribution from random and systematic errors are such that it is not valid to suggest other trends which, at first sight, may appear to be present. For example, the necessarily inexact nature of the reference signal which is subtracted from each particulate measurement will impose artificial trends (such as a peak at $80 \mathrm{kHz}$ ) in the experimental results.

An alternative method of measuring the attenuation in fluids is the spherical resonator. The error in the measurement of absorption using this technique has been estimated ${ }^{21}$ at $15 \%$, which is of similar magnitude to the error for the normalized attenuation with this technique for suspensions with concentrations above $1.0 \mathrm{~kg} / \mathrm{m}^{3}$. Another technique for measuring attenuation in suspensions, the Kramers-Kronig technique, ${ }^{22}$ is not applicable to the levels of attenuation observed in the suspensions under consideration here.

\section{CONCLUSION}

A series of reverberation tests performed on particulatefree water and water containing various concentrations of spherical glass beads has shown that the attenuation due to the particles is a readily measurable parameter using this technique. The measured attenuation agrees well with that predicted by theory for suspensions with a concentration greater than $0.5 \mathrm{~kg} / \mathrm{m}^{3}$ and improves as the difference in reverberation time increases.

\section{ACKNOWLEDGMENT}

This work is funded by DERA Winfrith (Contract No. SSDW3/0001) and their support is gratefully acknowledged.

${ }^{1}$ S. D. Richards, A. D. Heathershaw, and P. D. Thorne, "The effect of suspended particulate matter on sound attenuation in seawater,' J. Acoust. Soc. Am. 100, 1447-50 (1996).

${ }^{2}$ A. D. Heathershaw and P. D. Thorne, "Impact of suspended particulate matter on acoustic propagation in turbid coastal waters," in Proceedings of the International Maritime Defence Exhibition and Conference (Greenwich, London, 1995), Vol. 2, pp. 111-121.

${ }^{3}$ R. E. Francois and G. R. Garrison, "Sound absorption based on ocean measurements: Part I: Pure water and magnesium sulfate contributions," J. Acoust. Soc. Am. 72, 896-907 (1982).

${ }^{4}$ C. Devin, Jr., "Survey of thermal, radiation, and viscous damping of pulsating air bubbles in water," J. Acoust. Soc. Am. 31, 1654-1667 (1959).

${ }^{5}$ A. I. Eller, "Damping constants of pulsating bubbles," J. Acoust. Soc. Am. 47, 1469-1470 (1970).

${ }^{6}$ T. G. Leighton, The Acoustic Bubble (Academic, London, 1994), pp. 7883.

${ }^{7}$ S. D. Richards, “The effect of temperature, pressure and salinity on sound attenuation in turbid seawater," J. Acoust. Soc. Am. 103, 205-211 (1998).

${ }^{8}$ F. H. Fisher and V. P. Simmons, "Sound absorption in sea water," J. Acoust. Soc. Am. 62, 558-564 (1977).

${ }^{9}$ J. Lyman and R. H. Fleming, "Composition of sea water," J. Mar. Res. 3, 134-146 (1940)

${ }^{10}$ A. E. Hay and D. G. Mercer, "On the theory of sound scattering and viscous absorption in aqueous suspensions at medium and short wavelengths," J. Acoust. Soc. Am. 78, 1761-1771 (1985).

${ }^{11}$ J. Sheng and A. E. Hay, "An examination of the spherical scatterer approximation in aqueous suspensions of sand," J. Acoust. Soc. Am. 83, 598-610 (1988).

${ }^{12}$ R. J. Urick, "The absorption of sound in suspensions of irregular particles," J. Acoust. Soc. Am. 20, 283-289 (1948).

${ }^{13} \mathrm{G}$. Kurtze and K. Tamm, "Measurements of sound absorption in water and in aqueous solutions of electrolytes," Acustica 3, 33-48 (1953).

${ }^{14}$ N. R. Brown, T. G. Leighton, S. D. Richards, and A. D. Heathershaw, "'Sound absorption by suspended particulate matter," in High Frequency Acoustics in Shallow Water, edited by N. G. Pace, E. Pouliquen, O. Bergem, and A. P. Lyons (NATO SACLANT Undersea Research Centre, La Spezia, 1997), pp. 75-82.

${ }^{15}$ L. E. Kinsler, A. R. Frey, A. B. Coppens, and J. V. Sanders, Fundamentals of Acoustics (Wiley, New York, 1982), 3rd ed., p. 313.

${ }^{16}$ A. D. Pierce, Acoustics: An Introduction to its Physical Principles and Applications (McGraw-Hill, New York, 1981), p. 294.

${ }^{17}$ M. R. Schroeder, "New method of measuring reverberation time," J. Acoust. Soc. Am. 37, 409-412 (1965).

${ }^{18}$ L. E. Kinsler, A. R. Frey, A. B. Coppens, and J. V. Sanders, Ref. 15, p. 319.

${ }^{19}$ A. S. Ahuja, "Formulation of wave equation for calculating velocity of sound in suspensions," J. Acoust. Soc. Am. 51, 916-919 (1972).

${ }^{20}$ D. T. Noir and A. R. George, "Absorption of sound by homogeneous turbulence," J. Fluid Mech. 86, 593-608 (1978).

${ }^{21}$ P. D. Edmonds and J. Lamb, "A method for deriving the acoustic absorption coefficient of gases from measurement of the decay-time of a resonator,' Proc. Phys. Soc. London 71, 17-32 (1958).

${ }^{22}$ M. O'Donnell, E. T. Jaynes, and J. G. Miller, "Kramers-Kronig relationship between ultrasonic attenuation and phase velocity," J. Acoust. Soc. Am. 69, 696-701 (1981). 